\title{
Multisite Randomized Controlled Trial on the Provision of the EMDR Integrative Group Treatment Protocol for Ongoing Traumatic Stress Remote to Healthcare Professionals Working in Hospitals During the Covid-19 Pandemic
}

Pérez María Cristina ${ }^{1}$, Estévez María Elena ${ }^{2}$, Becker Yael $^{2}$, Osorio Amalia ${ }^{1}$, Jarero Ignacio ${ }^{2 *}$ and Givaudan Martha $^{2}$

${ }^{1}$ Department of Research, Ágape Desarrollo Integral, Mexico

${ }^{2}$ Department of Research, Mexican Association for Mental Health Support in Crisis, Mexico

Submission: September 27, 2020; Published: October 30, 2020

*Corresponding author: Ignacio Jarero, Department of Research, Mexican Association for Mental Health Support in Crisis, Mexico City, Mexico

\begin{abstract}
The aim of this longitudinal multisite randomized controlled trial (RCT), using a waitlist/delayed treatment control group design, was to evaluate the effectiveness of the Eye Movement Desensitization and Reprocessing-Integrative Group Treatment Protocol for Ongoing Traumatic Stress Remote (EMDR-IGTP-OTS-R) in reducing posttraumatic stress disorder (PTSD), depression, and anxiety symptoms in healthcare professionals working in hospitals during the Covid-19 pandemic in Puebla, Mexico. The study was conducted in 2020 in the city of Puebla, Mexico with healthcare professionals (nurses and medical doctors) working in ten hospitals who received Covid-19 patients. A total of 80 healthcare professionals met the inclusion criteria. Participants' ages ranged from 21 to 51 years old ( $M=33.24$ years). In this study, the early EMDR online group therapy intervention was initiated seven weeks after the first Covid-19 patients died in the hospitals. Intensive EMDR group treatment was provided. Data analysis by repeated measures ANOVA, showed that the EMDR-IGTP-OT-R had a significant effect on the explored variables (PTSD, Anxiety, and Depression) in time, group, and interaction time by group. Comparisons within means of the different pairs of longitudinal measurements were also useful to observe the consistency of the data. Results showed a large effect size (Cohen's d, from 1.93 to 1.25) between the pretest and the first posttreatment assessment, being able to attribute these effects to the EMDR-IGTP-OTS-R treatment. Results were confirmed with the between-group mean comparisons since the waitlist/delayed treatment act as a control group. In this case, the effect size (Cohen's d) varied from 3.95 to 1.92 showing a large effect of the difference between groups, attributed to the start time of the treatment. Results also showed an overall subjective global improvement in the study participants.

No adverse effects (e.g., symptoms of dissociation, fear, panic, freeze, shut down, collapse, fainting), or events (e.g., suicide ideation, suicide attempts, self-harm, homicidal ideation) were reported by the participants during treatment or at three months post-treatment follow-up while all participants were still working in their hospitals receiving COVID-19 patients. None of the participants showed clinically significant worsening/exacerbation of symptoms on the PCL-5 or HADS after treatment. To the best of our knowledge, this is the first RCT on early intervention with an evidence-based trauma-focus (TF) therapy (e.g., EMDR, TF-CBT) provided online in a group format and intensive treatment modality to healthcare professionals working in hospitals during the COVID-19 pandemic. This randomized controlled trial provides evidence for the effectiveness, efficacy, feasibility, and safety of the EMDR-IGTP-OTS-R in reducing posttraumatic stress, anxiety, and depression symptoms for healthcare professionals working in hospitals during the Covid-19 pandemic, expanding the EMDR therapy frontiers.
\end{abstract}

Keywords: Covid-19 healthcare professionals; Randomized Controlled Trial (RCT); Early EMDR online group therapy; Online group EMDR therapy; Eye movement desensitization and reprocessing (EMDR) online; EMDR-IGTP-OTS-Remote online; Longitudinal study; Posttraumatic stress disorder (PTSD); Anxiety; Depression.

\section{Introduction}

On December 30, 2019, a cluster of pneumonia cases of unknown etiology was reported in Wuhan, Hubei Province, China. On 9 January 2020, the China Center for Disease Control
(CDC) reported a novel coronavirus as the causative agent of this outbreak, which is phylogenetically in the SARS-CoV clade. On January 30, 2020, the World Health Organization (WHO) declared the emergence of the novel coronavirus as a public health 
emergency of international concern (PHEIC) [1]. On September 26, 2020, the World Health Organization (WHO) reported 32,429,965 confirmed COVID-19 cases with 985,823 deaths around the world and 715,457 confirmed cases with 75,439 deaths in Mexico, being the third country in the world with the highest number of deaths and the first country with the highest numbers of healthcare professionals' deaths [2,3]. The United Nations (UN) alerted the world about the high risk of a major mental health crisis caused by the COVID-19 pandemic and recommended the urgent widespread availability and use of mental health care and psychosocial support to address this situation [4].

The psychological impact on healthcare professionals working in hospitals with COVID-19 patients has been severe. In a study conducted in China by Liu et al., with a sample of 1,563 medical workers, results showed that $73.40 \%$ reported stressrelated symptoms, $\quad 50.70 \%$ depression symptoms, $44.70 \%$ anxiety, and $36.10 \%$ insomnia [5]. In another study conducted with 1,257 healthcare workers from 34 hospitals in China, results showed high rates of psychological stress: $50.4 \%$ had symptoms of depression, $44.6 \%$ symptoms of anxiety, $34 \%$ insomnia, and $71.5 \%$ general psychological distress. Regarding these outcomes, nurses, female staff, and staff working directly with patients were more likely to have severe scores [6]. In a study conducted in a New York City large medical center during a peak of inpatients admissions for COVID-19 with a sample of 657 healthcare professionals, $57 \%$ reported acute stress, $48 \%$ depression, and $33 \%$ anxiety symptoms [7]. Three hundred and thirty health professionals working in hospitals in the northern regions of Italy participated in an online survey. $71.2 \%$ had scores of state anxiety (the transitory state of fear and emotional tension as a response to a perceived threatening situation) above the clinical cutoff, $26.8 \%$ had clinical levels of depression, 31.3\% of anxiety, $34.3 \%$ of stress, and $36.7 \%$ of posttraumatic stress. Predictors of both emotional exhaustion and depersonalization (40.1\%) were: 1) female gender, and 2) being a nurse working in the hospital in contact with COVID-19 patients [8]. Healthcare frontline workers, nurses, and nursing students working with COVID-19 patients are experiencing significant levels of emotional distress and are exposed to a variety of sources of mental health burden $[9,10]$. Because of the increased risk for mental health challenges, to Pearman et al., COVID-19 may function as an occupational hazard for healthcare professionals and encourage efforts to intervene to provide relief now and in the future [11].

Transmissibility of COVID-19 via direct contact hinders faceto-face traditional mental health treatment options. Fortunately, Telehealth enables mental health professionals to deliver, support, or enhance mental health services while minimizing in-person contact. Telehealth is broadly defined as health services that are delivered via communication technologies, such as telephone and clinical video teleconferencing (CVT) which enables patients and mental health professionals to meet synchronously in real- time through a video platform [12]. The use of Telehealth has had an exponential increase during the COVID-19 pandemic. For example, online psychological counseling services, online mental health education, and online psychological self-help interventions have been widely used in mainland China, providing free $24-\mathrm{h}$ services on all days of the week [13]. Individuals with previous COVID-19 pandemic posttraumatic stress disorder (PTSD) may experience an increase in symptoms caused for new stressors. Also, new cases of PTSD may develop following exposure to COVID-19-related adverse experiences, especially in healthcare workers (e.g., prolonged exposure to a personal threat and patient's suffering and death). Therefore, the need for evidencebased online therapies to treat PTSD is crucial.

Telehealth has shown to be a promising treatment modality across a variety of trauma-exposed populations using cognitive behavioral therapy (CBT)-based interventions [14-16]. To Lewis et al., the efficacy of internet-delivered CBT for PTSD should be considered with caution [17]. Regarding online delivered EMDR therapy for PTSD, we conducted a systematic search for clinical trials and only found one published study. This uncontrolled open trial feasibility study examined the efficacy of a 6-week intervention, combining internet-delivered CBT with a web-based self-guided EMDR tool with 15 participants. In the first session, the clients were guided by an EMDR therapist by phone. Subsequent sessions were unguided. Although results showed potential efficacy, the study is limited by the lack of a control group, the small sample, the EMDR clinician minimal participation, and the unknown of the relative effects of each one of the two interventions (CBT and EMDR) [18].

\section{EMDR Therapy}

Eye Movement Desensitization and Reprocessing (EMDR) therapy is a structured, eight-phase comprehensive approach that addresses the past, present and future aspects of adverse life experiences, and it is guided by the Adaptive Information Processing (AIP) model [19]. This theoretical model posits that psychopathology is primarily caused by memories of traumatic or adverse life experiences that have been inadequately processed and maladaptively stored in a state-specific form. EMDR therapy and trauma-focused Cognitive Behavioral Therapy (TF-CBT) are recommended for the treatment of PTSD by the World Health Organization (WHO), the American Psychiatric Association (APA), the American Psychological Association (APA), the International Society for Traumatic Stress Studies (ISTSS), the National Institute for Health and Care Excellence (NICE), and the U.S. Department of Veterans Affairs/Department of Defense treatment guidelines (VA/DoD) [20-25]. EMDR therapy has shown efficacy as an early intervention treatment and on being the most cost-effective intervention for adults with PTSD among 11 evaluated options (e.g., TF-CBT, combined TF-CBT/SSRIs, combined somatic/ cognitive therapies, self-help with support, psychoeducation) $[26,27]$. 


\section{Psychology and Behavioral Science International Journal}

\section{EMDR-Integrative Group Treatment Protocol for On- going Traumatic Stress Remote}

The EMDR-integrative group treatment protocol (EMDR-IGTP) for early intervention was developed by members of the Mexican Association for Mental Health Support in Crisis (AMAMECRISIS) to deal with the extensive need for mental health services after Hurricane Pauline ravaged the coasts of the states of Oaxaca and Guerrero in the year 1997 [28]. It is the first EMDR individual treatment in a group format protocol. The protocol combines the eight EMDR treatment phases with a group therapy model and an art therapy format and uses the Butterfly Hug (BH) as a form of self-administered bilateral stimulation [29]. This protocol has a substantial body of research and has been used in its original format or with adaptations to suit the cultural circumstances, in numerous places around the world for thousands of survivors of natural or man-made disasters and with many other populations and circumstances (e.g, during ongoing war trauma, during an ongoing geopolitical crisis, with war refugee children, with children and adolescents who were victims of severe interpersonal violence, with cancer patients, with caregivers of patients with dementia, with refugee minors, as an emergency treatment to children survivors of terrorist attacks, with female survivors of exploitation, trafficking and early marriage) [30-58].

The risk of PTSD and comorbid disorders increases with the number of exposures. In contrast to studies of populations exposed to single-incident traumatic events, McFarlan et al. [59] mention that "patterns of emerging neurobiological dysregulation and symptom development with repeated trauma exposure have been demonstrated to occur on a continuum rather than as a sudden transition at the diagnosis threshold of full PTSD" (p. 9) [59]. In their clinical case conceptualization based on the AIP model, from a memory networks perspective, Jarero \& Artigas assert that for individuals living ongoing traumatic experiences (like healthcare professionals working in hospitals during the Covid-19 pandemic) in which there is not a post-trauma safety window for traumatic memory consolidation, the consolidation of the traumatic memory is prevented [60]. Therefore, the continuum of prolonged adverse experiences creates a cumulative trauma exposure memory network of linked pathogenic memories with similar emotional, somatic, sensorial, and cognitive information, that does not give the cumulative state-dependent traumatic memory network sufficient time to consolidate into an integrated whole [61-63]. They believe that this type of prolonged adverse experiences requires an especially designed EMDR treatment protocol [64-67]. Therefore, Jarero et al., adapted the EMDR-IGTP to treat older children, adolescents, and adults living with recent, present, or past prolonged adverse experiences (e.g., ongoing or prolonged traumatic stress) and developed the EMDR-IGTP for Ongoing Traumatic Stress (EMDR-IGTP-OTS) [68-71]. Due to the Covid-19 pandemic, and the impossibility to treat the clients in person, the EMDR-IGTP-OTS was adapted to be provided remotely (online) and the EMDR-IGTP-OTS-Remote was developed.

\section{Objective}

The objective of this longitudinal multisite randomized controlled trial (RCT) was to evaluate the effectiveness of the EMDR-IGTP-OTS-R in reducing posttraumatic stress disorder, depression, and anxiety symptoms in healthcare professionals working in ten hospitals who received COVID-19 patients during the pandemic in Puebla, Mexico.

\section{Method}

\section{Study Design}

To measure the effectiveness of the EMDR-IGTP-OTS-R on the dependent variables PTSD, Anxiety, and Depression, this study used a two arms longitudinal multisite randomized control trial (RCT) design. For ethical reasons (provide therapy to all participants), we selected a waitlist/delayed treatment control group design, comparing immediate treatment and waitlist/delayed treatment groups. PTSD, anxiety, and depression symptoms were measured in four-time points for all participants in the study: Time 1. Baseline assessment; Time 2. Immediate treatment group (ITG) post-treatment assessment; Time 3. Waitlist/delayed treatment control group (DTG) post-treatment assessment, and Time 4. Follow-up assessment. The subjective global improvement for all participants was measured at Time 4 Follow-up.

\section{Ethics and Research Quality}

The research protocol was reviewed and approved by the ten hospitals Institutional Review Boards (IRB): Hospital General de Traumatología y Ortopedia IMSS Puebla, Clínica 30 del IMSS, Hospital General la Margarita IMSS Puebla, Clínica 8 del IMSS, Unidad Médico Familiar del IMSS, Clínica 6 del IMSS, Hospital General de Zona No. 5 IMSS Metepec, Puebla; Hospital Regional de Zona No. 36 IMSS Puebla, Clínica 57 IMSS Puebla, Hospital General de Zona No. 1 "La Loma" IMSS Tlaxcala; in compliance with the International Committee of Medical Journal Editors recommendations, the Guidelines for Good Clinical Practice of the European Medicines Agency (version 1 December 2016) and the Helsinki Declaration as revised in 2013. The research quality of this study was based on the Consolidated Standards of Reporting Trials (CONSORT) 2010 Statement and the Standard Protocol Items Recommendation for Interventional Trials (SPIRIT) 2013 checklist [72-73].

\section{Participants}

This study was conducted in 2020 in the city of Puebla, Mexico with healthcare professionals (e.g., nurses, medical doctors) working in ten hospitals who received Covid-19 patients. 93 potential participants were recruited. Inclusion criteria were: (a) being adult, (b) being a healthcare professional (nurse or medical doctor) working in one of the ten hospitals who received Covid-19 patients, (c) voluntarily participating in the study, (d) not receiving specialized trauma therapy, (e) not receiving drug therapy for posttraumatic stress disorder symptoms. Exclusion criteria were: (a) ongoing self-harm/suicidal or homicidal 
ideation, (b) diagnosis of schizophrenia, psychotic or bipolar disorder, (c) diagnosis of dissociative disorder, (d) organic mental disorder, (e) a current, active chemical dependency problem, (f) significant cognitive impairment (e.g., severe intellectual disability, dementia), (g) presence of uncontrolled symptoms due to medical illness.

\section{Blind Randomization, Allocation Concealment Mechanism, and Blinding Procedure}

Simple randomization using a computer-generated random-number list with a 1:1 allocation ratio was used. Two independent assessors blind to treatment conditions conducted the randomization process to avoid allocation influence. One of them provided the random-number list and the other assigned random identification (ID) codes to each random-number in the list to protect the participant's identity. The treatment allocation sequence with the ID codes was concealed using sequentially numbered, opaque, sealed, and stapled envelopes. The safekeeping of the envelopes and the assignment of participants to each arm of the trial was overseen by a person not involved in the research study.
The treatment allocation of the participants was blinded for the research assistants (all mental health professionals) who conducted the intake interview and the enrollment. All the assessments, from Time 1 to Time 4, were conducted with the participants answering the self-administered instruments online and using an identification code instead of their name to protect their identity. An independent assessor received the instruments already answered online and was the safe keeper of all the data. There was no need to instruct participants to not reveal their treatment allocation to the persons conducting the assessments because all the participants answered the assessment instruments online.

Thirteen potential participants were excluded because they did not work in the ten hospitals as healthcare professionals (e.g, teachers, pharmacy employees). A total of 80 participants met the inclusion criteria. Participants' age ranged from 21 to 51 years old ( $M=33.24$ years old). Participation was voluntary with the participants' signed informed consent. There were 40 participants (39 female and 1 male) in the immediate treatment condition group and 40 participants (30 female and 10 male) in the waitlist/ delayed treatment condition group. See Flow Diagram.

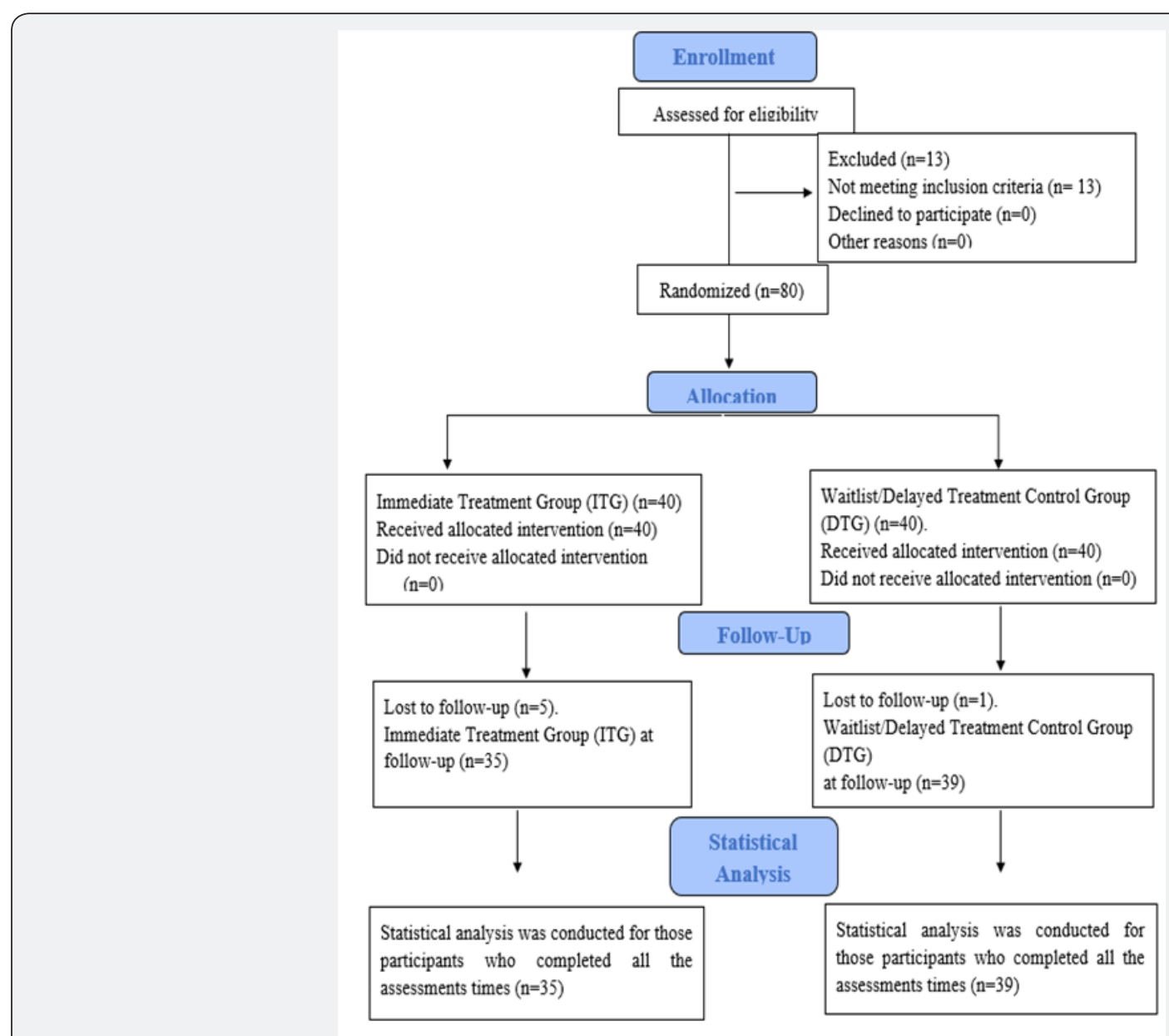

Flow Diagram 


\section{Instruments}

1)We used the Posttraumatic Stress Disorder Checklist for DSM-5 (PCL-5) provided directly by the National Center for PTSD (NCPTSD) and adapted, with the NCPTSD approval, the time interval for symptoms to be the past week instead of the past month [74-75]. The instrument was translated and backtranslated to Spanish. It contains 20 items, including three new PTSD symptoms (compared with the PTSD Checklist for DSM-IV) [76]: blame, negative emotions, and reckless or self-destructive behavior. Respondents indicate how much they have been bothered by each PTSD symptoms over the past week (rather than the past month), using a 5-point scale ranging from $0=$ not at all, $1=$ a little bit, $2=$ moderately, $3=$ quite a bit, and $4=$ extremely. A totalsymptoms score of zero to 80 can be obtained by summing the items. The sum of the scores yields a continuous measure of PTSD symptom severity for symptom clusters and the whole disorder. Psychometrics for the PCL-5, validated against the ClinicianAdministered PTSD Scale-5 (CAPS-5) diagnosis, suggest that a score of 31-33 is optimal to determine probable PTSD diagnosis [77-78], and a score of 33 is recommended for use at present. The PCL-5 is intended for a variety of clinical and research assessment tasks, including quantifying PTSD symptom severity, measuring the underlying construct of PTSD, establishing a provisional PTSD diagnosis, and estimating the presumed prevalence of PTSD. A score decrease between five to ten points demonstrates a clinically significant change. Decision rules for PTSD diagnosis based on PCL-5 are very accurate compared to CAPS diagnosis results. It is important to mention that at the first assessment, before answering the PCL-5, all participants were asked to focus specifically on the worst Covid-19 work-related event that currently bothered them the most; then at each subsequent assessment, they were asked to focus on the same event.

2) The Hospital Anxiety and Depression Scale (HADS) has been extensively used to evaluate these psychiatric comorbidities in various clinical settings at all levels of healthcare services and with the general population [79-80]. The instrument was translated and back-translated to Spanish. It is a 14 -item selfreport scale to measure the anxiety (7 items) and depression (7 items) of patients with both somatic and mental problems using a 4-point Likert scale ranging from 0 to 3. The response descriptors of all items are Yes, definitely (score 3); Yes, sometimes (score 2); No, not much (score 1); No, not at all (score 0). A higher score represents higher levels of anxiety and depression: a domain score of 11 or greater indicates anxiety or depression; 8-10 indicates borderline case; 7 or lower indicates no signs of anxiety or depression.

3) We used the Short PTSD Rating Interview Scale (SPRINT) to measure the subjective global improvement of the study participants at Time 4 Follow-up assessment. The SPRINT is an 8-item interview or self-rating questionnaire with solid psychometric properties that can serve as a reliable, valid, and homogeneous measurement of PTSD illness severity and global improvement as well as a measure of somatic distress; stress coping; and work, family, and social impairment [81]. The SPRINT contains two items to measure global improvement, one assessing percentage change and the other rating severity. Item 1: "How much better do you feel since beginning treatment? As a percentage between 0 and 100." Item 2: "How much have the above symptoms improved since starting treatment? 1 worse, 2 no change, 3 minimally, 4 much, 5 very much."

\section{Procedure}

\section{Enrolment, Assessments Times, Blind Data Collection, and Confidentiality of Data}

Each hospital sent an institutional email to their healthcare personnel (nurses and medical doctors) inviting them to participate in the study and giving them a phone number for the intake interview. Immediate treatment and waitlist/delayed treatment group participants completed the self-administered instruments online on an individual basis in the four different measurement moments. During time 1, research assistants (all mental health professionals) blind to treatment allocation, conducted the intake interview by phone, assessed potential participants for eligibility based on the inclusion/exclusion criteria, collected their data (e.g., name, age, gender, profession, email, telephone), obtained signed informed consent, enrolled participants in the study, sent the participant's data to the data safe keeper independent assessor and sent to the treatment allocation envelope's safe keeper only the participant's name and email.

After this procedure, the treatment allocation envelope's safe keeper (not involved in the research study) sent to each enrolled participant the link to answer the assessment instruments online, their ID codes, and the treatment dates. The data safe keeper independent assessor received the names, ID codes, and participant's allocation on each arm of the study from the envelope's safe keeper, and also the participant's instruments that were already answered online. Time 2 and Time 3 assessments were conducted online 15 days after the completion of each group treatment. Time 4 assessment was conducted online 90days after the waitlist/delayed group treatment' completion. All data was collected, stored, and handled in full compliance with the hospital's Institutional Review Board (IRB) requirements to ensure confidentiality. Each study participant gave their consent for access to their data, which was strictly required for study quality control. All persons involved in this research project were subject to professional confidentiality.

\section{Withdrawal from the Study}

All research participants had the right to withdraw from the study without justification at any time and with assurances of no prejudicial result. If participants decided to withdraw from the 
study, they were no longer followed up in the research protocol. There were six withdrawals for personal reasons not related to the study. See Flow Diagram.

\section{Treatment}

In this study, intensive early group EMDR online therapy was provided. Evidence suggests that more frequent scheduling of treatment sessions maximizes PTSD treatment outcomes, improves treatment response, and reduces treatment dropout [82-85]. Participants completed a total of four online group treatment sessions provided once a day during interspersed days (i.e., Monday, Wednesday, Friday, Sunday).

\section{Therapists and Treatment Fidelity}

EMDR-IGTP-OTS-R was provided online by 21 licensed EMDR clinicians formally trained in the protocol administration using a Zoom HIPAA Compliance program. Videos and screenshots of each online intervention were allowed and accepted by each participant in the informed consent and were used by independent raters to monitor the treatment fidelity and adherence to all steps of the protocol.

\section{EMDR-IGTP-OTS-R Treatment Description and Safety Measure}

The intake interview was made by phone for each potential group participant. Each of the participants received an average of 4.5 hours of online treatment, provided during four online group treatment sessions, once a day during four interspersed days. Treatment focused only on the distressing memories related to their work as healthcare professionals working in a hospital who received COVID-19 patients and did not address any other memories. During this process, participants followed directions from the team leader and worked quietly and independently on their distressing memories. The first treatment session lasted an average of 90 minutes. Subsequent treatment sessions lasted an average of 60 minutes. To encompass the whole ongoing traumatic stress spectrum lived by the participants, the team leader asked each of the participants to "Run a mental movie of everything that's happened in your work as a health professional, from right before the beginning of the first COVID-19 patient's death until today, or even looking into the future." The initial treatment target was the worst part of the mental movie. In subsequent sessions, the team leader asked participants to run the mental movie again and target any other memory that was currently disturbing, noticing associated emotions and body sensations. Participants in this study used the Butterfly Hug (BH) 24 times as a self-administered bilateral stimulation method to process traumatic material. All participants reprocessed more than one distressing memory. As a safety measure, participants were instructed to immediately report to the EMDR clinician's coordinator any adverse effects (e.g., symptoms of dissociation, fear, panic, freeze, shut down, collapse, fainting), events (e.g., suicide ideation, suicide attempts, self-harm, homicidal ideation), or symptoms worsening, during the entire study time-frame.

\section{Examples of Worst Experiences Reprocessed During the Online Treatment}

Generally, the worst experiences that the participants reprocessed were related to having to work with infected patients and fear of getting the virus themselves. In other cases, the worst experience was feeling like they had symptoms such as fever or headache and thinking that they might be sick and die or that they could infect a family member. Other participants reported that the worst experience was witnessing patients or co-workers suffer and die from the disease, causing fear and frustration. Some other experiences relate to not having adequate material or space to care for the large number of patients who showed up to the health centers and this made them feel helpless and angry. Also having to wear protective material, such as mouth covers and masks, for eight hours straight caused them physical injuries and they still had to continue using them. Some were afraid of losing their jobs.

\section{Statistical Analysis}

Statistical analysis was conducted for those participants, in both Immediate Treatment Group (ITG) $(\mathrm{N}=35)$ and Waitlist/ Delayed Treatment Control Group (DTG) (N=39), who completed all the four assessment times. Analyses of variance (ANOVA) for repeated measurements were used to analyze the effect of time and group for PTSD, Anxiety, and Depression; t-test and Cohen's $d$ effect size was calculated using within and between designs for mean comparison among the different measurements.

\section{Results}

\section{PTSD (PCL-5).}

Data analysis by repeated measures ANOVA revealed a significant effect for time $\left(\mathrm{F}(3,216)=594.54 \mathrm{p}<.001, \eta_{P}{ }^{2}=\right.$ .892), a significant effect for group ( $\mathrm{F}\left(1,72=26.57, \mathrm{p}<.001, \eta_{P}{ }^{2}\right.$ $=.270$ ) and a significant interaction between time and group, ( $F$ $\left.(3,216)=150.84, p<.001, \eta_{p}^{2}=.677\right)$. Comparison by group at base line did not show significant differences for time 1 . For time 2 significant differences between the Immediate Treatment Group (ITG) and the Waitlist/Delayed Treatment Control Group (DTG) were found, $\mathrm{t}(72)=-13.27, \mathrm{p}<.001, d=3.95$. Differences among groups continued over time. For time $3, \mathrm{t}(72)=-3.43 \mathrm{p}<.001$, $d=.79$, and for time $4, \mathrm{t}(72)=-2.48, \mathrm{p}<.01, d=.58$. In both ITG and DTG, mean scores showed a significant decrease after the first treatment session, $\mathrm{t}(34)=36.21, \mathrm{p}<.000, d=1.75$ (comparison between T1 and T2) for the ITG and t (38) = 37.24, p <.000, $d=$ 1.87 for the DTG (comparison between T2 and T3). There was also a significant decrease in the last following measurements in each group, $\mathrm{t}(34)=2.76, \mathrm{p}<.01, d=.111$ for ITG, and $\mathrm{t}(38)=5.47$ $\mathrm{p}<.001, d=.229$ for the DTG. In the DTG significant differences were also found between baseline and time $2, \mathrm{t}(38)=-3.0, \mathrm{p}<.005$, $d=.33$. Means showed an increase in PCL-5 scores for this group during the waiting time for treatment. See Figure 1 and Table 1. 


\section{Psychology and Behavioral Science International Journal}

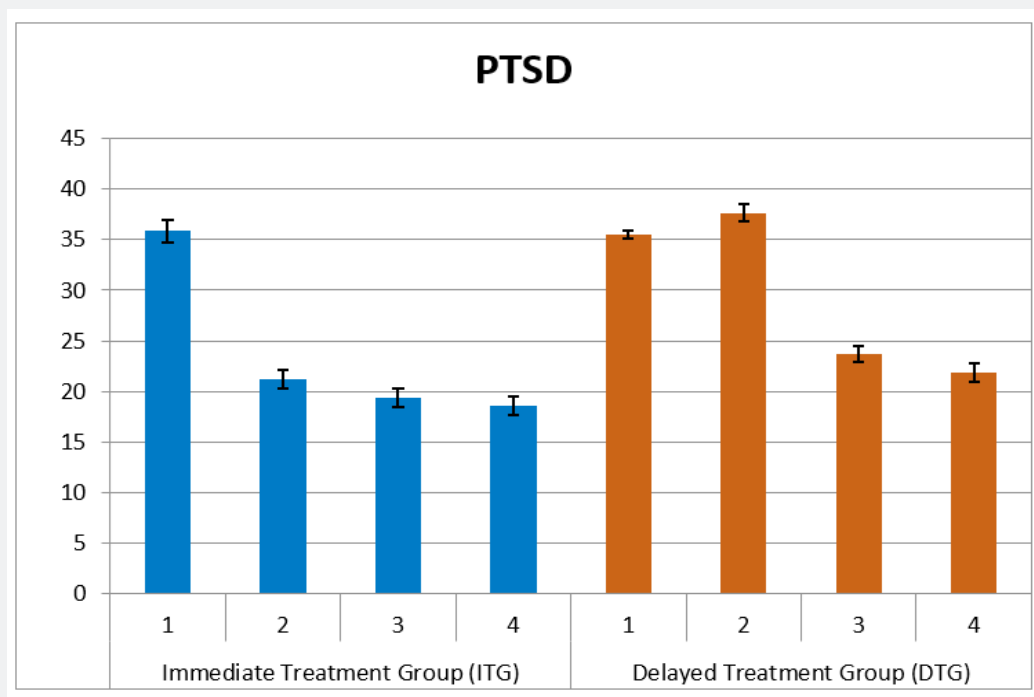

Figure 1: Mean scores and standard error for PTSD symptoms by time and group.

\section{Anxiety}

Repeated measures analysis of variance (ANOVA) for anxiety showed a significant effect for time $(F(3,216)=279.29, p<.001$, $\left.\eta_{p}^{2}=.795\right)$ and a significant effect for group $(\mathrm{F}(1,72)=11.01, \mathrm{p}$ $\left.<.001, \eta_{p}{ }^{2}=.133\right)$. Significant interaction effect was found for time and group $\left(\mathrm{F}(3,216)=47.72 \mathrm{p}<.001, \eta_{P}^{2}=.399\right.$. Mean comparison between groups did not show significant differences at baseline. For time 2, significant differences between groups were found, $\mathrm{t}$ $(72)=8.98, \mathrm{p}<.001, d=2.09$, which remain in time $3, \mathrm{t}(72)=2.54$ $\mathrm{p}<.01, d=.59$. No significant differences between groups were found by time 4 . Results analyzing differences by group show that for the ITG there were significant differences comparing baseline and time 2 , t (34) $=12.15, \mathrm{p}<.00, d=1.37$, as well as comparing time 2 and time $3, \mathrm{t}(34)=3.89, \mathrm{p}<.001, d=.25$. No differences were found between time 3 to time 4 . For the DTG no differences were found between base line and time 2 . Significant differences were found between time 2 and time 3 (after the first treatment session), $\mathrm{t}(38)=18.50, \mathrm{p}<.001, d=1.25$ and between time 3 and time $4, \mathrm{t}(38)=8.52, \mathrm{p}<.001, d=.59$. See Figure 2 and Table 1.

Table 1: Mean scores (M) and standard deviations (SD) for Immediate Treatment Group (ITG) and Delayed Treatment Group (DTG) on the baseline and follow up measurements.

\begin{tabular}{|c|c|c|c|c|c|c|c|c|}
\hline & \multicolumn{2}{|c|}{ Time 1} & \multicolumn{2}{|c|}{ Time 2} & \multicolumn{2}{|c|}{ Time 3} & \multicolumn{2}{|c|}{ Time 4} \\
\hline & $\mathbf{M}$ & SD & $\mathbf{M}$ & SD & $\mathbf{M}$ & SD & M & SD \\
\hline \multicolumn{9}{|c|}{ PTSD } \\
\hline ITG & 35.85 & 6.42 & 21.22 & 5.29 & 19.4 & 5.55 & 18.54 & 5.45 \\
\hline DTG & 35.48 & 3.64 & 37.64 & 5.32 & 23.69 & 5.21 & 21.87 & 5.99 \\
\hline \multicolumn{9}{|c|}{ Anxiety } \\
\hline ITG & 14.45 & 2.63 & 9.48 & 2.48 & 8.42 & 3.28 & 8.11 & 2.8 \\
\hline DTG & 14.15 & 2.68 & 14.28 & 2.1 & 9.89 & 1.42 & 8.66 & 1.53 \\
\hline \multicolumn{9}{|c|}{ Depression } \\
\hline ITG & 12.94 & 2.54 & 7.31 & 3.29 & 6.62 & 3.29 & 6.11 & 3.29 \\
\hline DTG & 12.71 & 2.58 & 12.84 & 2.43 & 7.58 & 1.81 & 6.3 & 1.68 \\
\hline
\end{tabular}




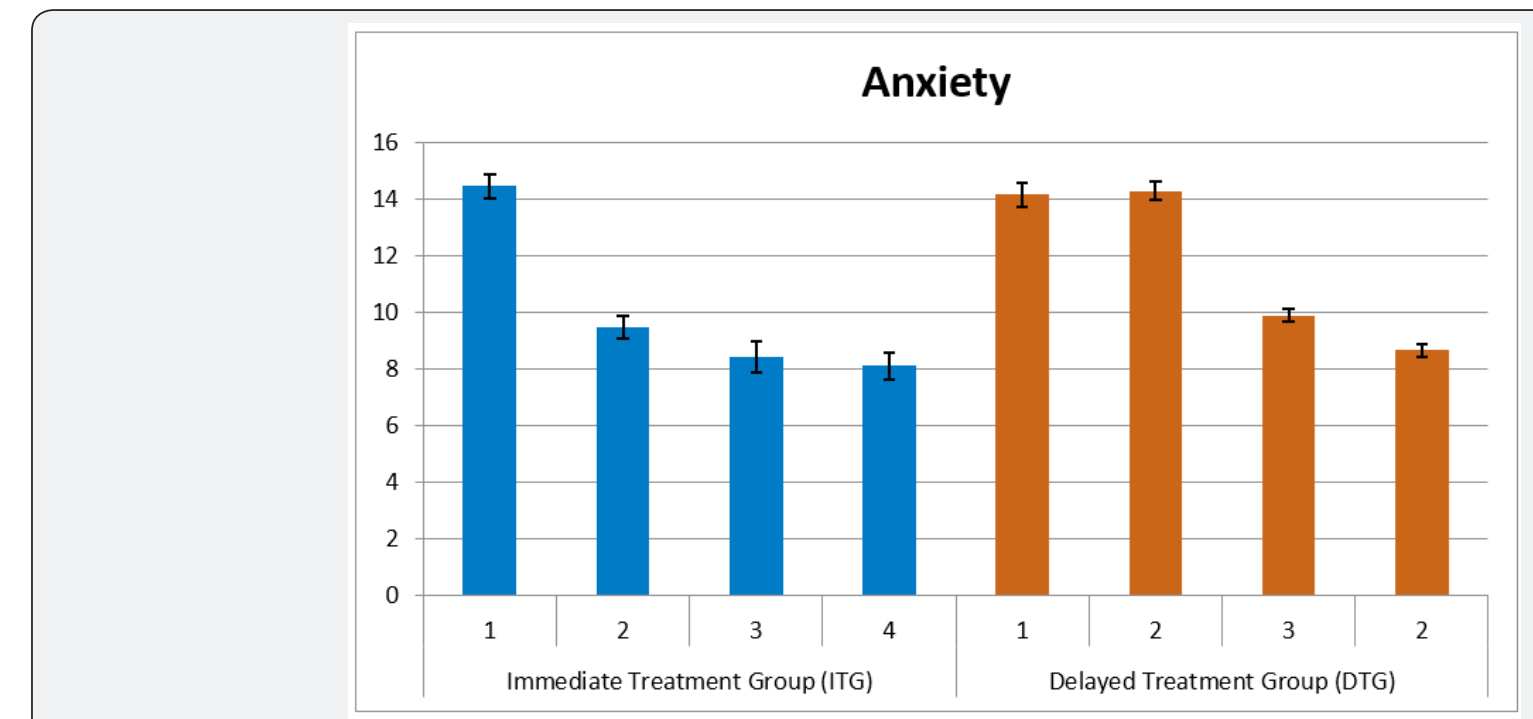

Figure 2: Mean scores and standard error for Anxiety by time and group.

\section{Depression}

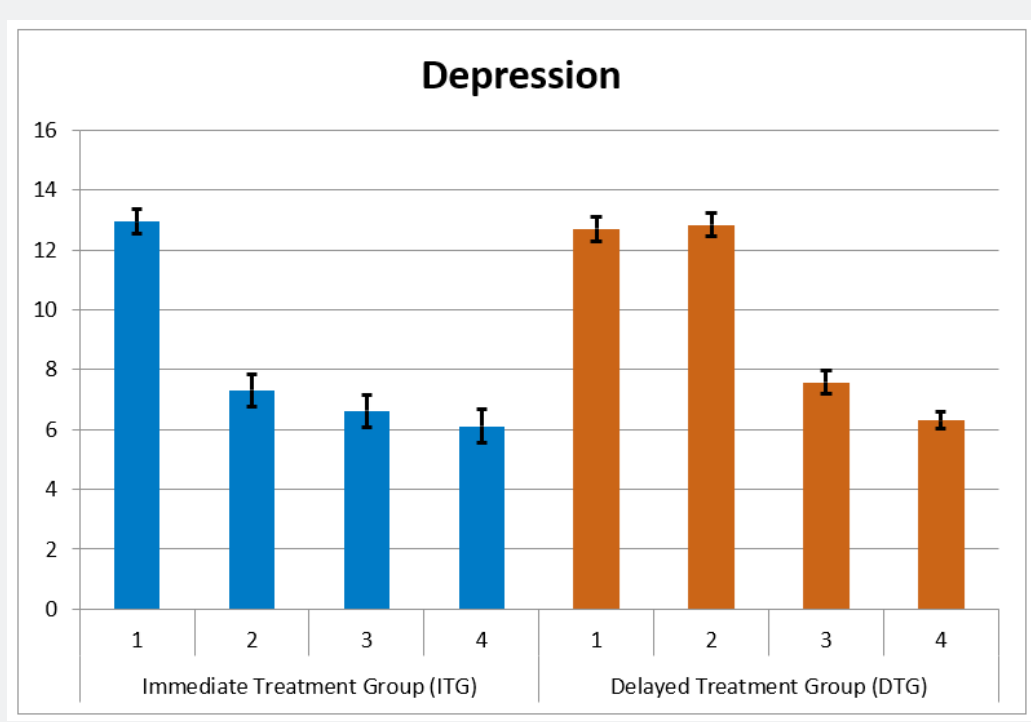

Figure 3: Mean scores and standard error for Depression by time and group.

About depression, significant effects were found through ANOVA repeated measurement analysis for time $(F(3,216)=$ 414.98, $\mathrm{p}<$. 001, $\eta_{P}^{2}=.852$ ). Results also showed significant interaction effects between time and group $(\mathrm{F}(3,216)=80.82$, $\left.\mathrm{p}<.001, \eta_{p}{ }^{2}=.529\right)$ and significant effects for group, $\mathrm{F}(1,72)=$ 8.18, $\mathrm{p}<.05, \eta_{P}^{2}=.102$. Comparison between groups did not showed significant differences at baseline. For time 2 significant differences were found between groups, $\mathrm{t}(72)=8.26, \mathrm{p}<.001, d=$ 1.92. No other intragroup differences were found in the following comparisons. For the ITG significant differences were found in all comparisons. Between baseline and time 1, t (34) = 14.55, $\mathrm{p}<.001$, $d=1.93$, between time 2 and time $3, \mathrm{t}(34)=4.08, \mathrm{p}<.001, d=.14$ and between time 3 and time 4, $\mathrm{t}(34)=4.09, \mathrm{p}<.001, d=.11$. For the DTG, no differences were found between base line and time 1. Significant differences were found between time 2 and time 3 , (after the first treatment session), $\mathrm{t}(38)=18.99, \mathrm{p}<.001, d=1.73$, and between time 3 and time $4, \mathrm{t}(38)=6.98, \mathrm{p}<.000, d=.51$. See Figure 3 and Table 1.

\section{Global Improvement}

Results of the Short PTSD Rating Interview Scale (SPRINT) showed that for item 1: "How much better do you feel since beginning treatment? The mean response at follow-up for the ITG was $84.6 \%(\mathrm{~N}=74)$ and for DTG it was $83.59 \%(\mathrm{~N}=74)$. About item 
2: "How much have the above symptoms improved since starting treatment? the mean response at follow-up for both groups was (4) much.

\section{Safety and symptoms worsening}

There were no adverse effects (e.g., symptoms of dissociation, fear, panic, freeze, shut down, collapse, fainting), or events (e.g., suicide ideation, suicide attempts, self-harm, homicidal ideation) reported by the participants during treatment or at three months post-treatment follow-up while all participants were still working in their hospitals receiving COVID-19 patients. None of the participants showed clinically significant worsening/exacerbation of symptoms on the PCL-5 or HADS scores after treatment.

\section{Discussion}

The aim of this longitudinal multisite randomized controlled trial (RCT) was to evaluate the effectiveness of the EMDR-IGTPOTS-R in reducing posttraumatic stress disorder, depression, and anxiety symptoms in healthcare professionals working in ten hospitals during the Covid-19 pandemic in Puebla, Mexico. A total of 80 healthcare professionals met the inclusion criteria and were randomly assigned to an immediate treatment group (ITG) or a deleted treatment group (DTG). Participants' ages ranged from 21 to 51 years old ( $\mathrm{M}=33.24$ years). Participation was voluntary with the participants' signed informed consent. In this study, the early EMDR online group therapy intervention was initiated seven weeks after the first Covid-19 patients died in the hospitals. Intensive EMDR group treatment was provided online by twentyone licensed EMDR clinicians formally trained in the protocol administration using a HIPAA Compliant Zoom program.

Analyses of variance for repeated measurements were used as well as t-test for between and within mean comparisons, including the calculation of Cohen's $d$ effect size. Results were consistent for the three studied variables (PTSD, anxiety, and depression). In all cases, significant effects for time, group, and interaction time by group were found. Comparisons within means of the different pairs of longitudinal measurements were also useful to observe the consistency of the data in the three included variables in both groups. Results showed a large effect size (Cohen's $d$, from 1.93 to 1.25) between the pretest and the first posttreatment evaluation, being able to attribute these effects to the EMDR-IGTP-OTS-R treatment. Results were confirmed with the between-group mean comparisons since the DTG act as a control group. In this case, the effect size (Cohen's $d$ ) varied from 3.95 for PTSD to 1.92 for depression showing, in all cases, a large effect of the difference between groups, attributed to the start time of the treatment. Specifically, it is interesting to note that there was a significant PTSD symptom's increment in the DTG between the first and the second assessments, reinforcing the recommendation for early EMDR treatment as soon as possible under these or similar circumstances. Time 4 assessment conducted online 90-days after the waitlist/delayed group treatment' completion showed a significant decrease in scores for PCL-5 in both groups denoting an effect of the treatment in PTSD symptoms reduction even though the study participant's continued working with infected patients, witnessing deaths, and facing their own and their families risk for the disease.

During the COVID-19 pandemic, front-line workers such as staff in administrative and logistic departments, emergency responders, medical technicians, and healthcare professionals (medical doctors and nurses) around the world, have been working in challenging environments (e.g., shortage of personal protective equipment's and medical supplies, longer working hours, risk of personal illness or death, fear of infecting their families), exposed to numerous stressors and tremendous pressure (e.g., choose which patients to save, exposure to the death and suffering of their patients), witnessing their colleagues dying, and suffering significant trauma with mental health implications in the short and long-term. Therefore, ensuring their mental health using evidence-based and cost-effective online therapies that can be delivered in group and individual formats is an ethical imperative. EMDR-IGTP-OTS-R can help upscale the Telehealth options and can reduce cultural resistance to treatment because it is minimally intrusive and does not require creating a narrative of the traumatic experience, verbal or written disclosure of details, the prolonged reliving of traumatic experiences, or homework relieving the adverse experience.

\section{Conclusion, Limitations, and Future Directions}

During the Covid-19 pandemic, healthcare professionals working in hospitals who received Covid-19 patients around the world presented posttraumatic stress, depression, and anxiety symptoms, among others (e.g., insomnia). The study results showed that the EMDR-IGTP-OTS-R can effectively and safely be provided online in a group format and in an intensive treatment modality to healthcare professionals living workrelated prolonged adverse experiences, to reduce PTSD, anxiety, and depression symptoms.

Besides the multiple strengths of this study, a limitation is the lack of a formal diagnosis of PTSD in the researched population and the 90-days follow-up. There is an imperative need to examine the effects of online-delivered EMDR therapy for PTSD in individual and group formats. We recommend randomized controlled trials using an instrument to conduct formal PTSD diagnosis (e.g., Clinician-Administered PTSD Scale-5), comparing EMDR therapy with other online-delivered therapies, and with follow-up at six or twelve months to evaluate the long-term treatment effects.

\section{Conflict of Interest and Founding}

The authors declare that they have no competing interests.

\section{Acknowledgments}

We want to express our gratitude to all the EMDR clinicians and research assistants that participated in this study, and especially to: Alma Belén Vergara Sánchez, Carlos Gancedo del Río, Araceli 
López Tlaque, Carmen María Priante Bretón, Carmen Rangel Calderón, Claudia Eugenia Tarasco Michel, Claudia Beatriz Varela Cabral, Carolina Domínguez Baron, Denisse Talamás Salazar, Edda Gabriela Pelayo Maurer, Giselle Ortiz Carreto, Graciela Verónica Guillén Sojo, Leticia Miranda Alamo, Marcela Obeso Fernández, Miryam Sandra del Carmen Jiménez Alarcón, Paola García Mier y Terán, Paula Ehlinger Escudero, Verónica Quintana Osorio, Viviana Triana, and Lynne Levinson.

\section{References}

1. Euro surveillance Editorial, T (2020) Note from the editors: World Health Organization declares novel coronavirus (2019-nCoV) sixth public health emergency of international concern. Euro Surveillance.

2. World Health Organization Coronavirus disease (COVID-19) Situation Report 115 (2020).

3. Forbes México (2020).

4. Policy Brief: COVID-19 and the Need for Action on Mental Health. (2020) United Nations.

5. Liu S, Yang L, Zhang C, Xiang YT, Liu Z, et al. (2020) Online mental health services in China during the COVID-19 outbreak. Lancet Psychiatry 7 : e17-e18.

6. Lai J, Ma S, Wang Y, Cai Z, Hu J, et al. (2020) Factors associated with mental health outcomes among health care workers exposed to coronavirus disease. JAMA Network Open 3: e203976.

7. Shechter A, Diaz F, Moise N, Anstey DE, Ye S, et al. (2020) Psychological distress, coping behaviors, and preferences for support among New York healthcare workers during the COVID-19 pandemic. Gen Hosp Psychiatry 66: 1-8.

8. Giusti EM, Pedroli E, D’Aniello GE, Stramba Badiale C, Pietrabissa G, et al. (2020) The Psychological Impact of the COVID-19 Outbreak on Health Professionals: Cross-sectional Study. Front Psychol 11: 1684.

9. Jackson D, Bradbury-Jones C, Baptiste D, Gelling L, Morin K., et al. (2020) Life in the pandemic: some reflections on nursing in the context of COVID-19. J Clin Nurs 29: 2041-2043.

10. Tomlin J, Dalgleish Warburton B, Lamph G (2020) Psychosocial Support for Healthcare Workers During the COVID-19 Pandemic. Front Psychol 11: 1960.

11. Pearman A, Hughes ML, Smith EL, Neupert SD (2020) Mental Health Challenges of United States Healthcare Professionals During COVID-19. Front Psychol 11: 2065

12. Brown FW (2017) Telepsychiatry and health technologies: A guide for mental health professionals. The American Journal of Psychiatry 174: $1126-1126$

13. Liu S, Yang L, Zhang C (2020) Online mental health services in China during the COVID-19 outbreak. Lancet Psychiatry 7: E17-E18.

14. Gros DF, Lancaster CL, López CM, Acierno R (2018) Treatment satisfaction of home-based telehealth versus in-person delivery of prolonged exposure for combat-related PTSD in veterans. Journal of Telemedicine and Telecare 24:51-55.

15. Morland LA, Mackintosh MA, Greene CJ, Rosen C, Chard K, et al. (2014) Cognitive processing therapy for posttraumatic stress disorder delivered to rural veterans via Tele Mental health: A randomized noninferiority clinical trial. Journal of Clinical Psychiatry 75: 470-476.

16. Morland LA, Mackintosh M, Rosen CS, Willis E, Resick P, et al. (2015)
Telemedicine vs. in-person delivery of cognitive processing therapy for women with posttraumatic stress disorder: A randomized noninferiority trial. Depression and Anxiety 32: 811-820.

17. Lewis C, Roberts NP, Simon N, Bethell A, Bisson JI (2019) Internetdelivered cognitive behavioral therapy for post-traumatic stress disorder: systematic review and meta-analysis. Acta Psychiatr Scand 140 (6): 508-521.

18. Spence J, Titov N, Johnston L, Dear BF, Wootton B, et al. (2013) Internet-delivered eye movement desensitization and reprocessing (iEMDR): an open trial. F1000research, 2.

19. Shapiro F (2018) Eye movements desensitization and reprocessing. Basic principles, protocols, and procedures ( $3^{\text {rd }}$ Edtn) Guilford Press, New York, United States.

20. Author (2013) Guidelines for the management of conditions that are specifically related to stress. World Health Organization, Geneva, Switzerland.

21. Guideline Watch (2009) Practice Guideline for the Treatment of Patients with Acute Stress Disorder and Posttraumatic Stress Disorder. American Psychiatric Association.

22. Clinical Practice Guideline for the Treatment of PTSD in Adults. (2017) American Psychological Association.

23. ISTSS Guidelines Committee (2019) Posttraumatic stress disorder prevention and treatment guidelines methodology and recommendations. Oakbrook Terrace.

24. National Institute for Health and Clinical Excellence (2018) Posttraumatic stress disorder. Evidence reviews on care pathways for adults, children and young people with PTSD.

25.VA/DoD Clinical Practice Guideline for the Management of Posttraumatic Stress Disorder and Acute Stress Disorder (2017)

26. Shapiro E, Maxfield L (2019) The Efficacy of EMDR Early Interventions. Journal of EMDR Practice and Research 13(4): 291-301.

27. Mavranezouli I, Megnin-Viggars O, Grey N, Bhutani G, Leach J, Daly C, et al. (2020) Cost effectiveness of psychological treatments for posttraumatic stress disorder in adults. PLoS ONE 15 (4): e0232245.

28. Jarero I, Artigas L (2009) EMDR integrative group treatment protocol. Journal of EMDR Practice \& Research 3(4): 287-288.

29. Artigas L, Jarero I, (2014) The Butterfly Hug. In M. Luber (Ed.) Implementing EMDR Early Mental Health Interventions for Man-Made and Natural Disasters .Springer, New York, United States pp.137-130.

30. Artigas L, Jarero I, Alcalá N, López Cano T (2014) The EMDR Integrative Group Treatment Protocol (IGTP) for children. In M. Luber (Ed.), Implementing EMDR early interventions for man-made and natural disasters. Springer, New York, United States pp.237-251.

31. Jarero I, Artigas L (2014) The EMDR Integrative Group Treatment Protocol (IGTP) for Adults. In: M Luber (Ed.) Implementing EMDR Early Mental Health Interventions for Man-Made and Natural Disasters Springer, New York, United States pp.253-265.

32. Adúriz ME, Knopfler C, Bluthgen C (2009) Helping child flood victims using group EMDR intervention in Argentina: Treatment outcome and gender differences. International Journal of Stress Management 16(2): 138-153.

33. Gelbach R, Davis K (2007) Disaster response: EMDR and family systems therapy under communitywide stress. In: F Shapiro, FW Kaslow, L Maxfield (Eds.), Handbook of EMDR and family therapy processes, Wiley, New York, United States pp.387-406. 
34. Maxfield L (2008) EMDR treatment of recent events and community disasters. Journal of EMDR Practice \& Research 2(2): 74-78.

35. Jarero I, Artigas L (2012) The EMDR Integrative Group Treatment Protocol: EMDR group treatment for early intervention following critical incidents. European Review of Applied Psychology 62: 219222.

36. Jarero I, Artigas L, Uribe S, Miranda A (2014) EMDR Therapy Humanitarian Trauma Recovery Interventions in Latin America and the Caribbean. Journal of EMDR Practice and Research 8(4): 260-268.

37. Jarero I, Artigas L, Hartung J (2006) EMDR integrative treatment protocol: A post-disaster trauma intervention for children \& adults. Traumatology 12: 121-129.

38. Adúriz ME, Salas C (2014) Aplicación del Protocolo Grupal e Integrativo con EMDR a las Víctimas de un Alud en Tartagal-Salta-Argentina. Revista Iberoamericana de Psicotraumatología y Disociación 6(2): 1-12.

39. Salas C (2014) Aplicación del Protocolo Grupal e Integrativo con EMDR a las Víctimas de un Alud en Angastaco-Argentina. Iberoamerican Journal of Psychotraumatology and Dissociation 6(2): 1-11.

40. Mehrotra S (2014) Humanitarian Projects and Growth of EMDR Therapy in Asia. Journal of EMDR Practice and Research 8(4): 252-259.

41. Jarero I, Artigas L, Montero M (2008) The EMDR integrative group treatment protocol: Application with child victims of a mass disaster. Journal of EMDR Practice and Research 2(2): 97-105.

42. Fernandez I, Gallinari E, Lorenzetti A (2003) A school-based intervention for children who witnessed the Pirelli building airplane crash in Milan, Italy. Journal of Brief Therapy 2(2): 129-136.

43. Maslovaric G, Zaccagnino M, Mezzaluna C, Perilli S, Trivellato D, et al. (2017) The Effectiveness of Eye Movement Desensitization and Reprocessing Integrative Group Protocol with Adolescent Survivors of the Central Italy Earthquake. Front Psychol 8: 1826.

44. Zaghrout Hodali M, Alissa F, Dodgson P (2008) Building resilience and dismantling fear: EMDR group protocol with children in an area of ongoing trauma. Journal of EMDR Practice and Research 2(2): 106113.

45. Jarero I, Artigas L (2010) EMDR integrative group treatment protocol: Application with adults during ongoing geopolitical crisis. Journal of EMDR Practice and Research 4(4): 148-155.

46. Allon M (2015) EMDR group therapy with women who were sexually assaulted in the Congo. Journal of EMDR Practice and Research 9(1): 28-34.

47. Jarero I, Roque-López S, Gómez J (2013) The Provision of an EMDRBased Multicomponent Trauma Treatment with Child Victims of Severe Interpersonal Trauma. Journal of EMDR Practice \& Research $7(1): 17-28$

48. Jarero I, Roque-López S, Gómez J, Givaudan M (2014a) Second Research Study on the Provision of the EMDR Integrative Group Treatment Protocol with Child Victims of Severe Interpersonal Violence. Iberoamerican Journal of Psychotraumatology and Dissociation 6(1): $1-24$.

49. Jarero I, Roque-López S, Gómez J, Givaudan M (2014b) Third Research Study on the Provision of the EMDR Integrative Group Treatment Protocol with Child Victims of Severe Interpersonal Violence. Iberoamerican Journal of Psychotraumatology and Dissociation 6(2): 1-22.

50. Jarero I, Rake G, Givaudan M (2017) EMDR Therapy Program for Advance Psychosocial Interventions Provided by Paraprofessionals.
Journal of EMDR Practice and Research 11(3): 122-128.

51. Passoni S, Curinga T, Toraldo A, Berlingeri M, Fernandez I, et al. (2018) Eye Movement Desensitization and Reprocessing Integrative Group Treatment Protocol (EMDR-IGTP) applied to caregivers of patients with dementia. Frontiers in Psychology 9: 967.

52. Harris H, Urdaneta V, Triana V, Vo CS, et al. (2018) A Pilot Study with Spanish-Speaking Latina Survivors of Domestic Violence Comparing EMDR \& TF-CBT Group Interventions. Open Journal of Social Sciences 6: 203-222

53. Hurn R Barron I (2018) The EMDR Integrative Group Treatment Protocol in a Psychosocial Program for Refugee Children: A Qualitative Pilot Study. Journal of EMDR Practice and Research 12(4): 208-223.

54. Perilli S, Giuliani A, Pagani M, Mazzoni GP, Maslovari G, et al. (2019) EMDR Group Treatment of Children Refugees -A Feasibility Field Study. Journal of EMDR Practice and Research 13(3): 143-155.

55. Smyth Dent KL, Fitzgerald J, Hagos Y (2019) A Field Study on the EMDR Integrative Group Treatment Protocol for Ongoing Traumatic Stress Provided to Adolescent Eritrean Refugees Living in Ethiopia. Psychology and Behavioral Science International Journal 12(4): 1-12.

56. Marie-Jo Brennstuhl, Fanny Bassan, Anne-Marie Fayard, Mathieu Fisselbrand, Amandine Guth, et al. (2019) Immediate treatment following the November 13 attacks: Use of an EMDR emergency protocol. European Journal of Trauma and Dissociation 3: 17-21.

57. Smyth-Dent K, Walsh SF, Smith S (2020) Field Study on the EMDR Integrative Group Treatment Protocol for Ongoing Traumatic Stress with Female Survivors of Exploitation, Trafficking and Early Marriage in Dhaka, Bangladesh. Psychology and Behavioral Science International Journal 15(3): 1-8.

58. Molero RJ, Jarero I, Givaudan M (2019) Longitudinal Multisite Randomized Controlled Trial on the Provision of the EMDR Integrative Group Treatment Protocol for Ongoing Traumatic Stress to Refugee Minors in Valencia, Spain. American Journal of Applied Psychology 8(4): 77-88.

59. McFarlane AC, Lawrence Wood E, Van Hooff M, Malhi GS, Yehuda $\mathrm{R}$ (2017) The need to take a staging approach to the biological mechanisms of PTSD and its treatment. Current Psychiatry Reports 19(2): 10 .

60. Jarero I, Artigas L (2018) AIP model-based Acute Trauma and Ongoing Traumatic Stress Theoretical Conceptualization. Iberoamerican Journal of Psychotraumatology and Dissociation 10(1): 1-7.

61. Jarero I, Amaya C, Givaudan M, Miranda A (2013) EMDR Individual Protocol for Paraprofessionals Use: A Randomized Controlled Trial Whit First Responders. Journal of EMDR Practice and Research 7(2): 55-64.

62. Centonze D, Siracusane A, Calabresi P, Bernardi G (2005) Removing pathogenic memories. Mol Neurobiol 32: 123-132.

63. van der Kolk BA, van der Hart O (1991) The intrusive past: The flexibility of memory and the engraving of trauma. American Imago 48(4): 425-454.

64. Jarero I, Uribe S, Artigas L, Givaudan M (2015) EMDR protocol for recent critical incidents: A randomized controlled trial in a technological disaster context. Journal of EMDR Practice and Research 9(4): 166173.

65. Jarero I, Schnaider S, Givaudan M (2019) EMDR Protocol for Recent Critical Incidents and Ongoing Traumatic Stress with First Responders: A Randomized Controlled Trial. Journal of EMDR Practice and Research 13(2). 
66. Encinas M, Osorio A, Jarero I, Givaudan M (2019) Randomized Controlled Clinical Trial on the Provision of the EMDR-PRECI to Family Caregivers of Patients with Autism Spectrum Disorder. Psychology and Behavioral Science International Journal 11(1): 1-8.

67. Estrada BD, Angulo BJ, Navarro ME, Jarero I, Sánchez-Armass 0 (in press) PTSD, Immunoglobulins, and Cortisol Changes after the Provision of the EMDR- PRECI to Females Patients with Cancer-Related PTSD Diagnosis. American Journal of Applied Psychology.

68. Jarero I, Artigas L, Uribe S, García LE, Cavazos MA, et al. (2015) Pilot Research Study on the Provision of the EMDR Integrative Group Treatment Protocol with Female Cancer Patients. Journal of EMDR Practice and Research 9(2): 98-105.

69. Jarero I, Artigas L, Uribe S, García LE (2016) The EMDR Integrative Group Treatment Protocol for Patients with Cancer. Journal of EMDR Practice and Research 10(3): 199-207.

70. Jarero I, Givaudan M, Osorio A (2018) Randomized Controlled Trial on the Provision of the EMDR Integrative Group Treatment Protocol Adapted for Ongoing Traumatic Stress to Patients with Cancer. Journal of EMDR Practice and Research 12(3): 94-104.

71. Osorio A, Pérez MC, Tirado SG, Jarero I, Givaudan M (2018) Randomized Controlled Trial on the EMDR Integrative Group Treatment Protocol for Ongoing Traumatic Stress with Adolescents and Young Adults Patients with Cancer. American Journal of Applied Psychology 7(4): 50-56.

72. Consolidated Standards of Reporting Trials (CONSORT) 2010 Statement (2010) BMJ 340: c869.

73. Standard Protocol Items Recommendation for Interventional Trials (SPIRIT) 2013 Checklist.

74. Weathers FW, Litz BT, Keane TM, Palmieri PA, Marx BP, et al. (2013) The PTSD Checklist for DSM-5 (PCL-5) Scale available from the National Center for PTSD.

75. Bovin MJ, Marx BP, Weathers FW, Gallagher MW, Rodriguez P, et al. (2016) Psychometric properties of the PTSD checklist for diagnostic and statistical manual of mental disorders- Firth edition (PCL-5) in veterans. Psychol Assess 28(11): 1379-1391.

76. American Psychiatric Association (2000) Diagnostic and statistical manual of mental disorders ( $4^{\text {th }}$ edtn) Text Revised) Arlington, Virginia.

77. Weathers FW, Blake DD, Schnurr PP, Kaloupek DG, Marx BP, et al. (2013a) Clinician-administered PTSD Scale for DSM-5. National Center for Posttraumatic Stress Disorder, Boston.

78. Franklin CL, Raines AM, Cucurullo LA, Chambliss JL, Maieritsch KP, et al. (2018) 27 ways to meet PTSD: Using the PTSD-checklist for DSM-5 to examine PTSD core criteria. Psychiatry Research 261: 504-507.

79. Zigmond AS, Snaith RP1 (1983) The Hospital Anxiety and Depression Scale. Acta Psychiatrica Scandinavica 67: 361-370.

80. Ying Lin C, Pakpour AH (2017) Using Hospital Anxiety and Depression Scale (HADS) on patients with epilepsy: Confirmatory factor analysis and Rasch models. Seizure (45): 42-46.

81. Connor KM, Davidson JRT (2001) SPRINT: A brief global assessment of post-traumatic stress disorder. International Clinical Psychopharmacology 16(5): 279-284.

82. Sciarrino N, Warnecke AJ, Ellen J Teng E (2020) A systematic review of intensive empirically supported treatments for posttraumatic stress disorder. Journal of Traumatic Stress 33(4): 443-454.

83. Hurley EC (2018) Effective Treatment of Veterans With PTSD: Comparison Between Intensive Daily and Weekly EMDR Approaches. Front Psychol 9: 1458.

84. Bongaerts H, Van Minnen A, de Jongh A (2017) Intensive EMDR to treat patients with complex posttraumatic stress disorder: A case series. Journal of EMDR Practice and Research 11(2): 84-95.

85. Gutner CA, Suvak MK, Sloan DM, Resick PA (2016) Does timing matter? Examining the impact of session timing on outcome. Journal of Consulting and Clinical Psychology 84: 1108-1115.

\section{Your next submission with Juniper Publishers will reach you the below assets}

- Quality Editorial service

- Swift Peer Review

- Reprints availability

- E-prints Service

- Manuscript Podcast for convenient understanding

- Global attainment for your research

- Manuscript accessibility in different formats

( Pdf, E-pub, Full Text, Audio)

- Unceasing customer service

Track the below URL for one-step submission https://juniperpublishers.com/online-submission.php 